\title{
Oxidative Stress in BPH
}

\author{
Savas $M,{ }^{1}$ Verit $A,{ }^{1}$ Ciftci $H,{ }^{1}$ Yeni E, ${ }^{1}$ Aktan E, ${ }^{1}$ Topal $U,{ }^{1}$ Erel $O^{2}$ \\ 'Urology Department, Medicine School of Harran University, Sanliurfa, Turkey, ${ }^{2}$ Biochemistry Department, Medicine School of Har- \\ ran University, Sanliurfa, Turkey
}

\section{ABSTRACT}

Introduction: In the present study, we investigated the relationship between potency of oxidative stress and $\mathrm{BPH}$ and this may assist to contribute to the realistic explanation of the ethiopathogenesis of $\mathrm{BPH}$.

Methods: Seventy four newly diagnosed men with BPH (mean age: $54 \pm 11.2$ ), who had not undergone any previous treatment for $\mathrm{BPH}$, and 62 healthy volunteers (mean age: 55 \pm 14 ) were enrolled in the present study. To determine the antioxidative status of plasma, total antioxidant capacity (TAC) was calculated, and to determine the oxidative status of plasma (TOS) total peroxide levels were measured. The ratio of TAC to total peroxide was accepted as an indicator of oxidative stress (OSI). Data are presented as mean SD \pm unless specified. Student $t$-test and correlation analyses were used to evaluate the statistical significance differences in the median values recorded for all parameters between $\mathrm{BPH}$ and control group.

Results: Plasma TAC TOS were found in patients and controls $(1.70 \pm 0.32,1.68 \pm 0.19 \mu$ mol Trolox Equiv./L), $(12.48 \pm 1.98,12.40 \pm 1.14 \mu \mathrm{mol} / \mathrm{L})$ respectively. OSI was calculated as $7.57 \pm 1.91,7.48$ \pm 1.33 , respectively. Plasma TAC, TOS and OSI levels were not found to be significantly difference between patients and control subjects $(p>0.05, p>0.05, p>0.05)$.

Conclusions: The present study has shown that there were not relationship between potency of oxidative stress and BPH. Further well designed studies should be planned to find out whether the oxidative stress-related parameters play role in $\mathrm{BPH}$ as an interesting pathology in regard of the etiopathogenesis.

Keywords: benign prostatic hyperplasia, oxidative stress, prostate

\section{INTRODUCTION}

Benign prostatic hyperplasia (BPH) is a highly prevalent disease of oldermencaused by nonmalignant, unregulated

Running Title: Oxidative Stress in BPH growth of the prostate gland. ${ }^{1}$ The etiology of $\mathrm{BPH}$ is not well understood. Androgens, essential for normal prostate growth and development, play a prominent role. However, there is also evidence that metabolic

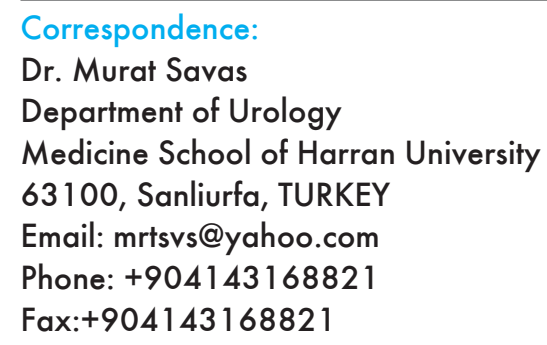


disturbances may promote prostate hyperplasia and $\mathrm{BPH}$ pathogenesis. Reactive oxygen species (ROS) are produced in metabolic and physiological processes, and harmful oxidative reactions may occur in organisms which remove ROS via enzymatic and nonenzymatic antioxidative mechanisms. Oxidative stress, which has been implicated in over 100 disorders, develops in consequence..$^{2,3}$ To our knowledge, there has been no report involving the oxidative/antioxidative status of plasma in the BPH. In the present study, we investigated the relationship between potency of oxidative stress and $\mathrm{BPH}$ and this may assist to contribute to the realistic explanation of the ethiopathogenesis of $\mathrm{BPH}$.

\section{METHODS}

A prospective study was carried out at Urology Department of Harran University Medical School Hospital from Jan 15, 2008 to April 31, 2008. Seventy four newly diagnosed men with BPH (mean age: $54 \pm 11.2$ years), who had not undergone any previous treatment for $\mathrm{BPH}$, and 62 healthy volunteers (mean age: $55 \pm 14$ years) were enrolled in the present study after giving informed consent for participation in the study. The study protocol conforms to the principles of the Declaration of Helsinki and was approved by the institutional ethics review board. Past medical history and current medications were recorded, in addition to detailed physical examination in all cases. Exclusion of criteria was the presence of liver dysfunction, diabetes mellitus, heart failure or renal failure, inflammatory or infection diseases, smoking and oral antioxidant supplementation at the moment of the enrollment and any signs of malignancy. None of the subjects had a drinking habit, and none of them had consumed any alchol prior to starting blood collection. All subjects body mass index was defined as the weight in kilograms divided by the height in metres squared $\left(\mathrm{kg} / \mathrm{m}^{2}\right)$. Blood samples were withdrawn into heparinized tubes from a cubital vein after overnight fasting and immediately stored in ice. Plasma was separated from cells by centrifugation at $3000 \mathrm{rpm}$ for $10 \mathrm{~min}$. Plasma samples were stored at $-80^{\circ} \mathrm{C}$ until analysis. TAC of plasma was determined using a novel automated measurement method developed by Erel ${ }^{7}$. In this method, hydroxyl radicals, which are the most potent biological radicals, are produced. In this assay, the antioxidative effect of the sample against the potent free radical reactions, which are initiated by hydroxyl radical that was produced, is measured. The assay has excellent precision values, which are lower than $3 \%$. The results are expressed as millimoles of Trolox equivalent per liter. Total peroxide concentrations of plasma samples were determined using the ferrous oxidation in xylenol orange, version 2 (FOX2) methods with minor modifications. The FOX2 test system is based on the oxidation of ferrous ion to ferric ion by various types of peroxides contained within the plasma samples, to produce a colored ferric-xylenol orange complex whose absorbance can be measured. The FOX2 reagent was prepared by dissolving ammonium ferrous sulphate $(9.8 \mathrm{mg})$ in 250 $\mathrm{mM} \mathrm{H} 2 \mathrm{SO} 4(10 \mathrm{ml})$ to give a final concentration of $250 \mathrm{mM}$ ferrous ion in acid. This solution was then added to $90 \mathrm{ml}$ HPLC-grade methanol containing 79.2 mg butylated hydroxytoluene (BHT). Finally, $7.6 \mathrm{mg}$ xylenol orange was added with stirring to make the final working reagent $(250 \mathrm{mM}$ ammonium ferrous sulphate, $100 \mathrm{mM}$ xylenol orange, $25 \mathrm{mM} \mathrm{H} 2 \mathrm{SO} 4$, and $4 \mathrm{mM} \mathrm{BHT}$ in $90 \% \mathrm{vol} / \mathrm{vol}$ methanol in a final volume of $100 \mathrm{ml}$ ). The blank working reagent contained only ferrous sulphate. Aliquots $(200 \mathrm{~mL})$ of plasma were mixed with $1800 \mathrm{~mL}$ FOX2 reagent. After incubation at room temperature for $30 \mathrm{~min}$, the vials were centrifuged at $12000 \mathrm{~g}$ for 10 minutes. Absorbance of the supernatant was then determined at $560 \mathrm{~nm}$. Total peroxide content of plasma samples was determined as a function of the absorbance difference between test and blank tubes using a solution of $\mathrm{H} 2 \mathrm{O} 2$ as standard. The coefficient of variation for individual plasma samples was less than $5 \% .^{4}$ The percent ratio of total peroxide level to TAC was accepted as oxidative stress index (OSI). To perform the calculation, the result unit of TAC, mill moles of Trolox equivalent per liter, was changed to micromoles of Trolox equivalent per liter, and OSI was calculated with the formula; OSI = Total peroxide, $\mu \mathrm{mol} /$ liter / TAC, $\mu \mathrm{mol}$ Trolox equivalent/L $x 100$. Data are presented as mean \pm SD. Student $t-$ test and correlation analyses were used to evaluate the statistical significance differences in the median values recorded for all parameters between $\mathrm{BPH}$ and control group. Statistical significance was defined as $\mathrm{P}<0.05$.

\section{RESULTS}

Socioeconomic status was similar in both groups $(P>0.05)$. Demographic characteristics of the subjects are shown in Table 1. There were no significant differences in age and body mass index between patients and the control subjects $(P>0.05)$. Total PSA and f-PSA levels were significantly higher in patients than control subjects $(P=0,001)$ (Table 2). Plasma TAC TOS were found in patients and controls $(1.70 \pm 0.32$, $1.68 \pm 0.19 \mu \mathrm{mol}$ Trolox Equiv./L), $(12,48 \pm 1.98$, $12.40 \pm 1.14 \mu \mathrm{mol} / \mathrm{L}$ ), respectively. OSI was calculated as $7.57 \pm 1.91,7.48 \pm 1.33$, respectively. Plasma TAC, was not found to be significantly difference between patients and control subjects $(P>0.05)$. Total antioxidant capacity (TAC, $\mu \mathrm{mol}$ Trolox Equiv./L) difference in the patients and control groups was shown in the Figure 1. Plasma TOS and OSI levels were not found to be significantly difference between patients and control subjects $(P>0.05)$ (Table 1). Total peroxide 
(total oxidative status (TOS,) ( $\mu \mathrm{mol} / \mathrm{L}$ ) difference in the patients and control groubs and the relationship between OSI (Total peroxide, $\mu \mathrm{mol} / \mathrm{liter} / \mathrm{TAC}, \mu \mathrm{mol}$ Trolox equivalent/L $\times 100)(A U)$ in the patients and control subjects (Figure 2,3).

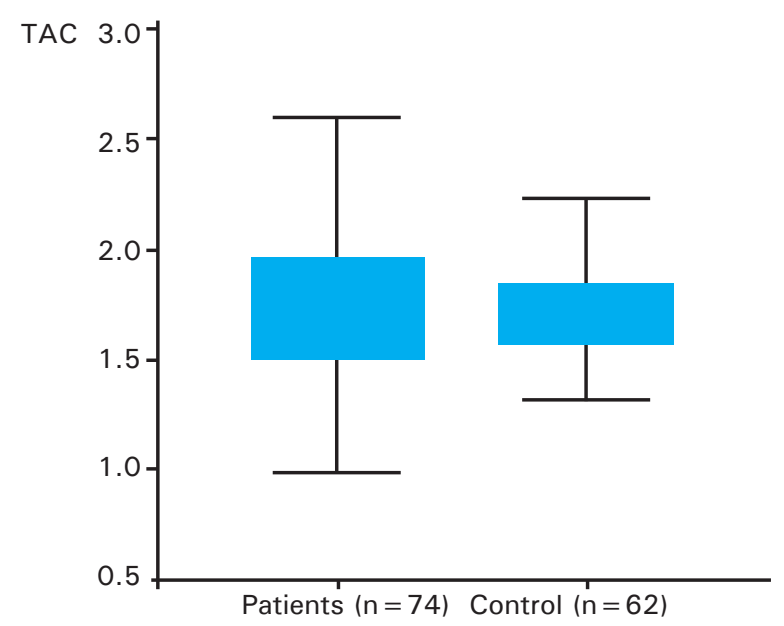

Figure 1. Total antioxidant capacity (TAC, $\mu \mathrm{mol}$ Trolox Equiv./L ) difference in the patients and control groups

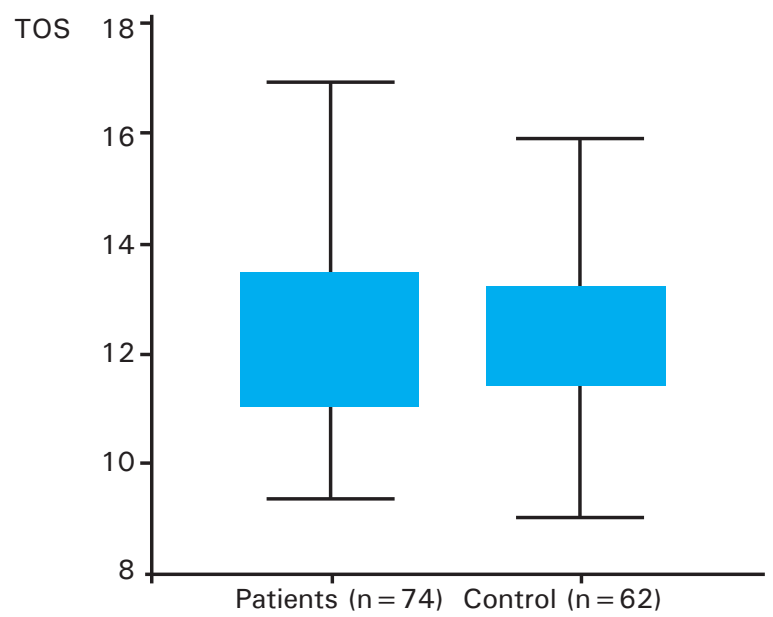

Figure 2. Total peroxide (total oxidative status (TOS,) ( $\mu \mathrm{mol} / \mathrm{L})$ ) difference in the patients and control groubs

\section{DISCUSSION}

In our study we determined plasma TAC, total peroxide levels and $\mathrm{OSI}$ in the patients with $\mathrm{BPH}$ not significantly increased $(p>0.05)$. The etiology of $\mathrm{BPH}$ is not well understood. Androgens, essential for normal prostate growth and development, play a prominent role. However, there is also evidence that metabolic disturbances may promote prostate hyperplasia and $\mathrm{BPH}$ pathogenesis. BPH and prostate cancer are diseases associated with aging. ${ }^{5}$ Aging is an inherently complex process that is manifested within an organism at genetic, molecular, cellular, organ, and system levels. Although the fundamental mechanisms are still poorly understood, a growing body of evidence points toward reactive oxygen species (ROS) as one of the primary determinants of aging. ${ }^{6}$

Reactive oxygen species (ROS) are produced in metabolic and physiological processes, and harmful oxidative reactions may occur in organisms which remove ROS via enzymatic and nonenzymatic antioxidative mechanisms. Under some conditions increases in oxidants and decreases in antioxidants cannot be prevented, and the oxidative/antioxidative balance shifts towards the oxidative status. Oxidative stress has been implicated in the ethiopathology of prostate cancer and $\mathrm{BPH}$. It has been demonstrated that oxidative stress is positively correlated with prostate cancer. ${ }^{7}$ The relationship between BPH and oxidative stress has not been fully understood yet. Blood contains many antioxidant molecules that prevent and/or inhibit harmful free radical reactions. Free radicals and oxidants such as superoxide radical anions, hydroxyl radicals and hydrogen peroxide are produced in metabolic and physiological processes. The oxidative effects of free radicals are controlled by exogenous antioxidants, such as vitamins $E$ and $C$, and also by endogenous antioxidants, such as scavenger enzymes superoxide dismutase, glutathione peroxidase and catalase, as well as albumin, biluribin and uric acid. Under some conditions, increases in oxidants and

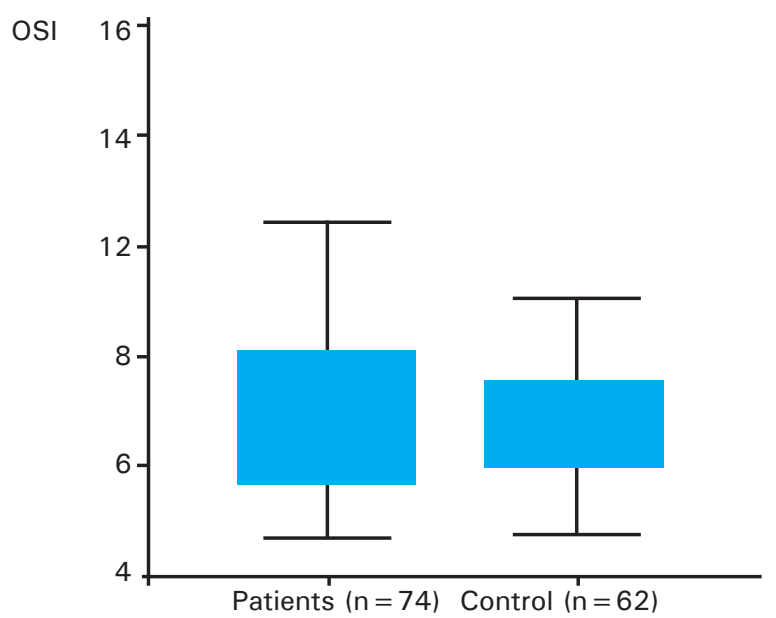

Figure 3. The relationship between OSI (Total peroxide, $\mu \mathrm{mol} /$ liter / TAC, $\mu \mathrm{mol}$ Trolox equivalent/L $\mathbf{x} 100)(A U)$ in the patients and control subjects 
Table1. Demographic characteristics of patients with BPH and control subjects and comparison of $t-P S A, f-P S A$, TAC, TOS and OSI parameters in the patient and control groups

\begin{tabular}{lccc}
\hline Parameters & Control $(\boldsymbol{n}=\mathbf{6 2})$ & Patient $(\mathbf{n}=\mathbf{7 4})$ & $\boldsymbol{P}$ \\
\hline Age (years) & $55 \pm 14$ & $54 \pm 11.12$ & $>0.05$ \\
BMI $\left(\mathrm{kg} / \mathrm{m}^{2}\right)$ & $24.3 \pm 4.4$ & $23 \pm 5.1$ & $>0.05$ \\
t-PSA (ngr/ml) & $1.02 \pm 0.98$ & $3.90 \pm 3.31$ & 0.001 \\
f-PSA (ngr/ml) & $0.29 \pm 0.25$ & $1.13 \pm 1.10$ & 0.001 \\
TAC (mmol Trolox Eqv./L) & $1.68 \pm 0.19$ & $1.70 \pm 0.32$ & 0.74 \\
TOS ( $\mu \mathrm{mol} / \mathrm{L})$ & $12.40 \pm 1.14$ & $12.48 \pm 1.98$ & 0.96 \\
OSi (AU) & $7.48 \pm 1.33$ & $7.57 \pm 1.91$ & 0.76 \\
\hline
\end{tabular}

Data are presented as mean \pm SD, BMI: Body mass index, t-PSA: Total prostate specific antigen, f-PSA: Free prostate specific antigen TAC: Total antioxidant capacity; TOS: Total oxidative status; OSI: Oxidative stress index

decreases in antioxidants can not be prevented, and the oxidative-antioxidative balance shifts toward the oxidative stress. Plasma concentrations of antioxidants can be measured separately in the laboratory, but these measurements are time-consuming, labor intensive and costly. Because the antioxidative effects of components the antioxidants of plasma are additive, the measurement of total antioxidant capacity (TAC) reflects the antioxidative status of the plasma. ${ }^{8}$

We evaluated the total antioxidative status of plasma using a more recently developed measurement method by Erel. ${ }^{9}$ In this method, TAC against especially potent radical reactions, which strongly lead to oxidative damage of biomolecules such as lipids, proteins and DNA, is measured. Hydrogen peroxide and the other derivates of peroxides, produced physiologically and increased in some conditions, diffuse into plasma, where they are overwhelmed by antioxidant components of plasma and are simultaneously consumed. We evaluated the total oxidative status of plasma by measuring the total peroxide level. To our knowledge, there has been no report involving the oxidative/antioxidative status of plasma in the $\mathrm{BPH}$. In the present study, we investigated the relationship between potency of oxidative stress and $\mathrm{BPH}$ and this may assist to contribute to the realistic explanation of the ethiopathogenesis of $\mathrm{BPH}$.

Lipid peroxidation is one parameter of the oxidants in the plasma and increased lipid peroxidation has been well documented in the peripheral blood of patient's plays etiology of many diseases. Demirbag et al have shown that plasma TAC was lower and OSI and total peroxide levels using a more recently developed measurement method by Erel were higher in patients with NHYA classIII/ IV than NHYA class I/II. ${ }^{4}$ Harma et al also found that TAC was decreased and the total peroxide level increased in patients with complete hydatidiform mole (CHM) using developed method by Erel. ${ }^{10}$ Aydin et al. have found elevated lipid peroxidation in the prostate cancer group versus controls and the BPH group. ${ }^{7}$ However, DogruAbbasoglu et al. have found no significant change in lipid peroxidation or antioxidant system parameters in the plasma of patients with $\mathrm{BPH}$ and prostate cancer. ${ }^{5}$ As parallel with Dogru-Abbasoglu et al. we found that total peroxide levels were not significantly higher in the patients with BPH than in control subjects using a more recently developed measurement method by Erel. Androgens, essential for normal prostate growth and development, play a prominent role. However, there is also evidence that metabolic disturbances may promote prostate hyperplasia and BPH pathogenesis. Previous etiological models of BPH have focused primarily on the role of sex steroid hormones. Both androgens and estrogens may stimulate prostate growth. Adipose tissue, which accumulates with age, aromatizes circulating testosterone into estrogen, and it has been hypothesized that alterations in the balance between testosterone and estrogen levels in prostate tissue with age may contribute to $\mathrm{BPH} .{ }^{11,12}$

An alternative mechanism for BPH may be related to metabolic disturbances. Obesity and elevated fasting glucose are components of the metabolic syndrome both obesity and the metabolic syndrome are associated with systemic inflammation and oxidative stress. ${ }^{13,14}$ Inflammation has been implicated as a primary stimulus for prostate carcinogenesis, and it is possible that $\mathrm{BPH}$ represents an alternate, nonmalignant pathway of unregulated prostate growth promoted by oxidative stress, inflammatory mediators, and IGFs. ${ }^{15,16}$ Indeed, analyses of surgical specimens have shown that $\mathrm{BPH}$ is usually associated with inflammation and that the extent and severity of the inflammation corresponds to the amount of prostate enlargement. ${ }^{17}$

$\mathrm{BPH}$ is a phenomenon followed by an age-dependent increase in volume of the prostate throughout the entire life of a man. The growth and involution of the prostate depend on the quantitative relationship between the 
rate of cell proliferation and cell death. Most previous studies have focused on proliferation and apoptotic rates in benign hyperplastic human prostates, Siegfried et al. reported that there is an increase in proliferation rate and decrease in apoptotic rate in benign hyperplastic prostate tissue. ${ }^{18}$ It has been clearly established that ROS and ROS-modulated molecules participate in both intrinsic and extrinsic apoptotic pathways. ${ }^{19}$ While oxidative stress induces the apoptosis, apoptosis reduce in $\mathrm{BPH}$. In this study although we did not evaluate the apoptosis, any change in oxidative stress in our patients supported the results of above mentioned studies. In addition, we suggest the comparison of TOS in elders and young groups to determine the effect of oxidative stress on ageing for the further studies. In this present study we only assayed the TAC, TOS and OSI levels in the similar age groups as study and control.

\section{CONCLUSION}

The present study has shown that there were not relationship between potency of oxidative stress and $\mathrm{BPH}$. Further well designed studies should be planned to find out whether the oxidative stress-related parameters play role in $\mathrm{BPH}$ as an interesting pathology in regard of the etiopathogenesis. Literature widely concerns the concepts of ageing, oxidative stress, apoptosis and $\mathrm{BPH}$ which seem to have possible relationships.

\section{REFERENCES}

1. Roehrborn CG, McConnell JD. Etiology, pathophysiology, epidemiology, and natural history of benign prostatic hyperplasia. In: Walsh PC, Retik AB, Wein AW, Vaugh E, eds. Campbell's Urology. Philadelphia: Lipincott Williams and Wilkins 2002; 1297-1336.

2. Harma M, Harma M, Erel O. Oxidative stress in women with preeclampsia. Am J Obstet Gynecol 2005; 192(2):656-7.

3. Yeni E, Gulum M, Selek S, Erel O, Unal D, Verit A et al. Comparison of oxidative/antioxidative status of penil corpus cavernosum blood and peripheral venous blood. Int J Impot Res 2005; 17(1):19-22.

4. Demirbag R, Yilmaz R, Erel O, Gultekin U, Asci D, Elbasan $Z$. The relationship between potency of oxidative stress and severity of dilated cardiomyopathy. Can J Cardiol 2005; 21(10):851-5.

5. Dogru-Abbasoglu SJ Aykac-Toker G, Kocak T, Unluer E, Uysal M. Antioxidant enzyme activities and lipid peroxides in the plasma of patients with benign prostatic hyperplasia or prostate cancer are not predictive. J Cancer Res Clin Oncol 1999; 125(7):402-4.

6. Kevin C. Kregel and Hannah J. Zhang. An integrated view of oxidative stress in aging: basic mechanisms, functional effects, and pathological considerations. Am J Physiol Regul Integr Comp Physiol 2007; 292: R18-R36.

7. Aydin A, Arsova-Sarafinovsca Zorica, Sayal A, Eken A, Erdem O. Oxidative stress and antioxidant status in nonmetastatic prostate cancer and benign prostatic hyperplasia. Clinical Biochemistry 2006; 39:176-179.

8. Berges RR, Vucanovic J, Epstein JI ,Walsh PC, Isaacs JT. Implication of cell cycle kinetic changes during the progression of human prostate cancer. Clin Cancer Res 1995; 1: 473-479.

9. Erel O. A novel automated method to measure total antioxidant response against potent free radical reactions. Clin Biochem 2004; 37:112-9.

10. Harma M, Harma M, Erel O. Increased oxidative stres in patients with hydatiform mole. Swiss Med Wkly 2003; 133:563-6.
11. Monti S, Di Silverio F, Lanzara S, Varasano P, Martini C, TostiCroce $\mathrm{C}$ et al. Insulin-like growth factor-I and -II in human benign prostatic hyperplasia: relationship with binding proteins 2 and 3 and androgens. Steroids 1998; 63:362-366.

12. Shibata Y, Ito K, Suzuki K, Nakano K, Fukabori Y, Suzuki R, et al. Changes in the endocrine environment of the human prostate transition zone with aging: simultaneous quantitative analysis of prostatic sex steroids and comparison with human prostatic histological composition. Prostate 2000; 2:45-55.

13. Haffner S, Taegtmeyer H. Epidemic obesity and the metabolic syndrome. Circulation 2003; 108:1541-1545.

14. Furukawa S, Fujita T, Shimabukuro M, Iwaki M, Yamada Y, Nakajima $Y$, et al. Increased oxidative stress in obesity and its impact on metabolic syndrome. J Clin Invest 2004; 114:17521761.

15. Nelson WG, De Marzo AM, Isaacs WB. Prostate cancer. N Engl J Med 2003; 349:366-381.

16. Wang W, Bergh A, Damber JE. Chronic inflammation in benign prostate hyperplasia is associated with focal upregulation of cyclooxygenase-2, Bcl-2, and cell proliferation in the glandular epithelium. Prostate 2004; 61:60-72.

17. Di Silverio F, Gentile V, De Matteis A, Mariotti G, Giuseppe V, Luigi PA et al. Distribution of inflammation, pre-malignant lesions, incidental carcinoma in histologically confirmed benign prostatic hyperplasia: a retrospective analysis. Eur Urol 2003; 43:164-175.

18. Siegfried C, Wrenger $M$, Senge $T$, Schulze $H$. Immunhistochemical determination of age related proliferation rates in normal and benign hyperplastic human prostates. Urol Res 1993; 21: 305308.

19. Matsuzawa A, Ichijo H. Stress-responsive protein kinases in redox-regulated apoptosis signaling. Antioxid Redox Signal $2005 ; 7: 472-481$. 